\title{
Investigating which variables from the core outcome variables in juvenile idiopathic arthritis (JIA) are the best predictors of classification as a responder to treatment with methotrexate (MTX)
}

\author{
SP Hirani*1, L Kassoumeri², K Mulligan ${ }^{1}$, Collaborators PRINTO3, \\ N Ruperto ${ }^{3}$, $\mathrm{PWoO}^{4}$, LR Wedderburn ${ }^{2}$ and SP Newman ${ }^{1}$
}

Address: ${ }^{2}$ UCL Behavioural Medicine, London, UK, ${ }^{2}$ UCL Institute of Child Health, London, UK, ${ }^{3}$ Paediatric Rheumatology International Trials Organisation, Genova, Italy and ${ }^{4}$ UCL Centre of Paediatric and Adolescent Rheumatology, London, UK

* Corresponding author

\author{
from I5th Paediatric Rheumatology European Society (PreS) Congress \\ London, UK. 14-17 September 2008 \\ Published: 15 September 2008 \\ Pediatric Rheumatology 2008, 6(SuppI I):PI20 doi:10.I I86/I546-0096-6-SI-PI20
}

This abstract is available from: http://www.ped-rheum.com/content/6/SI/PI20

(c) 2008 Hirani et al; licensee BioMed Central Ltd.

\section{Background}

Percentage change scores over six core outcome variables (COV) are utilised to classify response to treatment in JIA. This study aimed to determine the relative contribution of each of the COV towards the classification of improvement following treatment, at the ACR30, 50 and 70 levels.

\section{Methods}

Using a dataset of 410 JIA patients treated with MTX, (provided by PRINTO) 3 sets of logistic regression analyses were conducted, one at each classification level, to determine the likelihood of a classification of improvement. For each level a series of univariate logistic analyses were conducted to identify, from the six COV, individually significant $(\mathrm{p}<0.05)$ predictors of improvement. These variables were entered into a stepwise multivariate logistic analysis to identify independent predictors of classification $(\mathrm{p}<0.01)$ at each level.

\section{Results}

For all three classification criteria, each core variable change score was an individually significant predictor of improvement as classified using classical procedures. Within multivariate analyses, physician global rating was the 'best' indicator of improvement at all criteria levels. ESR did not contribute significantly above other variables in all the multivariate analyses. The remaining variables all contributed significantly in the $30 \%$ and $50 \%$ models. The limited variable models showed relatively good classification of cases, $93.9 \%, 89 \%$, and $87.3 \%$ at the $30 \%$, $50 \%$ and $70 \%$ levels respectively.

\section{Conclusion}

Individually all the core variables are important indicators of improvement in JIA. However, when used in combination a limited set of variables can lead to a relatively accurate indication of response to treatment by JIA patients. 